Case Report

\title{
Low-Dose Tolvaptan for the Treatment of Dilutional Hyponatremia in Cirrhosis: A Case Report and Literature Review
}

\author{
Guo Shen ${ }^{1}$ and Hainv Gao ${ }^{2}$ \\ ${ }^{1}$ The First Hospital of Zhuji, 122nd Huansha Nan Road, Zhuji 311800, China \\ ${ }^{2}$ The State Key Laboratory of Diagnosis and Treatment for Infectious Disease, College of Medicine, The First Affiliated Hospital, \\ Zhejiang University, 79th Qingchun Road, Hangzhou 310003, China \\ Correspondence should be addressed to Hainv Gao; gaohainv@163.com
}

Received 7 January 2014; Revised 3 April 2014; Accepted 15 April 2014; Published 30 April 2014

Academic Editor: Mario Pirisi

Copyright (c) 2014 G. Shen and H. Gao. This is an open access article distributed under the Creative Commons Attribution License, which permits unrestricted use, distribution, and reproduction in any medium, provided the original work is properly cited.

\begin{abstract}
Dilutional hyponatremia is common in decompensated cirrhosis and can be successfully treated by tolvaptan, a vasopressin V2receptor antagonist. Data were lacking regarding the effects of tolvaptan on cirrhotic patients with a Child-Pugh score of $>10$ and a serum sodium concentration of $<120 \mathrm{mmol} / \mathrm{L}$. We report a case of forties man with a 20 -year history of chronic hepatitis B presenting with yellow urine and skin. Laboratory tests demonstrated prolonged prothrombin time, markedly elevated total bilirubin, severe hyponatremia, and a Child-Pugh score of $>10$. The patient was diagnosed with dilutional hyponatremia and was treated with recommended dosage tolvaptan at first. The serum concentration of sodium recover but the patient felt obviously thirsty. As the dosage of tolvaptan was decreased accordingly from $15 \mathrm{mg}$ to $5 \mathrm{mg}$, the patient still maintained the ideal concentration of serum sodium. This case emphasizes that cirrhotic patient with higher Child-Pugh scores and serum sodium concentration of $<120 \mathrm{mmol} / \mathrm{L}$ can be treated with lower dose of tolvaptan.
\end{abstract}

\section{Introduction}

Dilutional hyponatremia is defined as a serum sodium concentration of $<135 \mathrm{mmol} / \mathrm{L}$. This condition is common in decompensated cirrhosis, with an incidence as high as $49 \%$ [1]. Recent studies have shown that hyponatremia is not only a sign of disease severity but also a direct factor that could aggravate the disease. In addition, acute hyponatremia is considered as an independent predictor of mortality in patients with cirrhosis $[2,3]$. However, traditional therapies such as fluid restriction and supplementation of high-dose sodium show little effect. A prospective randomized study showed that only $0-26 \%$ of patients treated with water restriction had serum sodium concentrations of $>5 \mathrm{mmol} / \mathrm{L}$ [3]. Patients feel thirsty and have difficulty in finishing water restriction therapy too. The efficacy of high-dose sodium replacement is limited and the replacement can aggravate ascites and edema as well. Therefore, sodium administration is not recommended for treatment of dilutional hyponatremia in cirrhotic patients [4]. Recently, it was reported that tolvaptan, a vasopressin V2-receptor antagonist, had significant effect on the therapy of hyponatremia in cirrhotic patients. Nevertheless, few data are available regarding the effects of tolvaptan on cirrhotic patients with a Child-Pugh score of $>10$ and a serum sodium concentration of $<120 \mathrm{mmol} / \mathrm{L}$. Herein, we present a case of a decompensated cirrhotic patient with a serum sodium concentration of $117 \mathrm{mmol} / \mathrm{L}$ who received low-dose tolvaptan.

\section{Case Presentation}

A 47-year-old man with a 20-year history of chronic hepatitis B was hospitalized with the complaint of yellow urine and skin for 5 months. The patient had been treated with lamivudine in combination with adefovir for 1 year and discontinued treatment of his own 10 months ago. Five months ago, he developed the symptoms of yellow urine and skin and followed by being diagnosed as decompensated hepatitis cirrhosis B and treated with entecavir. During entecavir treatment, he had a total bilirubin concentration of $200-350 \mu \mathrm{mol} / \mathrm{L}$, prothrombin time of $20-31 \mathrm{~s}$, and 
TABLE 1: Serum sodium concentrations and urine volumes of the present patient under the treatment of different doses of tolvaptan.

\begin{tabular}{|c|c|c|c|c|c|c|c|c|c|c|c|c|c|c|c|}
\hline Data (day) & Baseline $^{*}$ & 1 & 2 & 3 & 4 & 5 & 8 & 11 & 14 & 17 & 20 & 30 & 60 & 120 & $1(\mathrm{yr})$ \\
\hline Dosage of tolvaptan (mg) & NA & 15 & 15 & 7.5 & 7.5 & 5 & 5 & 5 & 5 & 5 & 5 & & $\mathrm{NA}$ & NA & NA \\
\hline Urine volume $(\mathrm{mL})$ & 2050 & 4400 & 6100 & 4050 & 4200 & 3100 & 2750 & 3000 & 3550 & 3800 & 2750 & 4300 & 4000 & NA & NA \\
\hline Serum sodium $(\mathrm{mmol} / \mathrm{L})$ & 132 & 117 & 123 & 130 & 130 & 128 & NA & 128 & NA & 127 & NA & 129 & 135 & NA & 138 \\
\hline Total bilirubin $(\mu \mathrm{mol} / \mathrm{L})$ & 317 & 244 & NA & 246 & NA & 227 & NA & 196 & NA & 199 & NA & 157 & 92 & 38 & 35 \\
\hline $\operatorname{ALT}(\mathrm{U} / \mathrm{L})$ & 52 & 51 & NA & NA & 44 & NA & NA & 34 & 30 & 28 & NA & 33 & 17 & 20 & 17 \\
\hline $\mathrm{PT}(\mathrm{s})$ & 27.3 & 23.2 & NA & NA & 25 & NA & NA & 24.1 & 24.9 & 23.8 & NA & 21.8 & 22.4 & 19.6 & 14.2 \\
\hline $\mathrm{BP}(\mathrm{mmHg})$ & $124 / 69$ & $114 / 64$ & NA & NA & NA & NA & $107 / 62$ & $104 / 72$ & $114 / 68$ & NA & $122 / 71$ & $115 / 65$ & $127 / 70$ & NA & $125 / 65$ \\
\hline Body weight (Kg) & 76 & 67.5 & 65 & 66.5 & 66.5 & 69 & NA & NA & 65.5 & NA & 61 & 61.5 & NA & NA & 71 \\
\hline Child-Pugh score & 14 & 14 & NA & NA & NA & NA & NA & $\mathrm{NA}$ & $\mathrm{NA}$ & NA & NA & 11 & 10 & 7 & 5 \\
\hline
\end{tabular}

* Baseline: the data was from the first day when the patient was admitted.

a Child-Pugh score of $>10$. The bilirubin level was persistently high despite repeat (up to four times) artificial liver support therapy. Meanwhile, during the course of treatment, the patient showed hepatic encephalopathy, a large amount of ascites, and hyponatremia with numerous serum sodium concentrations as low as $115 \mathrm{mmol} / \mathrm{L}$. He received large dosage of diuretic, lower salt intake (less than 2 grams per day), water restriction to less than $500 \mathrm{~mL}$ per day, and one time paracentesis, but the symptom was not relieved. The patient was treated with tolvaptan at an initial dose of $15 \mathrm{mg}$ qd. After therapy, he had a urine volume of $6100 \mathrm{~mL}$ and became obviously thirsty, although the serum sodium concentration showed significant recovery. The tolvaptan dosage was decreased to $7.5 \mathrm{mg} \mathrm{qd}$, but he remained thirsty. For the large volume of urine and the obvious thirst, the dosage of diuretic was decreased once but resumed soon because of increasing ascites. Finally, the dosage of tolvaptan was maintained at $5 \mathrm{mg} \mathrm{qd}$, and the patient's serum sodium concentration was consistently $128 \mathrm{mmol} / \mathrm{L}$ and daily urine volume was between 3000 and $4000 \mathrm{~mL}$ (see Table 1). During the course of therapy, the condition of patient was markedly improved, as indicated by the continuously decreased level of total bilirubin. After 1 month of treatment with tolvaptan, the drug was stopped and the serum sodium concentration was maintained at $130 \mathrm{mmol} / \mathrm{L}$. Coused drugs were entecavir tablet, ademetionine injection, zolpidem tartrate tablet, lactulose, magnesium isoglycyrrhizinate injection, albumin injection, piperacillin-tazobactam injection, and ornithine aspartate injection during the therapy of tolvaptan. The patient was discharged from the hospital with a total bilirubin concentration as low as $94 \mu \mathrm{mol} / \mathrm{L}$ and a serum sodium concentration of $135 \mathrm{mmol} / \mathrm{L}$. From that time, the patient stopped diuretics therapy and just used entecavir. At the 3-month follow-up, total bilirubin concentration was only $38 \mu \mathrm{mol} / \mathrm{L}$ and serum sodium concentration was $137 \mathrm{mmol} / \mathrm{L}$. $\mathrm{B}$ ultrasound showed small amount of ascites. At the 1-year follow-up, the liver function of the patient was normal and B ultrasound showed no ascites at all.

\section{Discussion}

We presented a middle-aged man with a history of 20-year chronic hepatitis B who presented with yellow urine and skin.
Laboratory tests demonstrated prolonged prothrombin time, markedly elevated total bilirubin, severe hyponatremia, and a Child-Pugh score of $>10$. The patient was diagnosed with dilutional hyponatremia and was successfully treated with low-dose tolvaptan.

Dilutional hyponatremia is the most common complication of cirrhosis. The Study of Ascending Levels of Tolvaptan in Hyponatremia 1 and 2 (SALT-1 and SALT-2) trials demonstrated that when tolvaptan was used at the dosage between 15 and $60 \mathrm{mg} / \mathrm{d}$ for 30 days, serum sodium concentrations can be resumed in most patients. In addition, the most common adverse event was thirst, and rapid correction of the serum sodium concentration did not occur [5]. However, it should be noted that only 63 patients with cirrhosis were enrolled in these 2 trials and those with a Child-Pugh score of $>10$ or a serum sodium concentration of $<120 \mathrm{mmol} / \mathrm{L}$ were excluded. Thus, more clinical experience is required to investigate the effects of tolvaptan on cirrhotic patients with Child-Pugh scores of $>10$.

Recently, several studies have evaluated the effects of tolvaptan at lower doses for the treatment of cirrhosis. In a double-blind, parallel-group, multicenter phase III clinical trial in Japan [6] which aimed to verify the efficacy of low-dose tolvaptan in patients with liver cirrhosis-associated ascites and insufficient response to conventional diuretic treatment and investigate its pharmacokinetic and pharmacodynamic profiles, a total of 40 patients with cirrhosis and an average concentration of initial serum sodium for all patients being $>120 \mathrm{mmol} / \mathrm{L}$ were included. 20 patients belonged to Child-Pugh Class C. The results showed that tolvaptan at a dose of $7.5 \mathrm{mg} / \mathrm{d}$ could increase the urine output and decrease the ascitic volume. The serum sodium concentrations were increased significantly on the first day. In our case, low-dose of tolvaptan was successfully used to treat a patient with an initial serum sodium concentration as low as $117 \mathrm{mmol} / \mathrm{L}$ and a Child-Pugh score of $>10$. Tolvaptan showed good safety since the blood pressure of patient was in the normal range during the therapy.

There are several possible reasons for the successful treatment of hyponatremia in cirrhosis with low-dose of tolvaptan. Firstly, it is reported that $99 \%$ of tolvaptan molecules bind to plasma proteins after entry into the bloodstream. For 
patients with cirrhosis, the serum albumin concentration was low due to decreased protein synthesis, which could result in a reduced protein-tolvaptan binding rate and an increase in free tolvaptan plasma concentration. Moreover, albumin levels vary in healthy individuals by $10 \%$ [7], which could lead to altered drug efficacy. Secondly, tolvaptan is primarily metabolized by CYP3A4 and the activity of CYP3A4 enzymes is changed in cirrhotic patients [8]. Thirdly, portal-systemic shunting in patients with advanced cirrhosis could reduce the first-pass effect of drugs and lead to a significant increase in absorption. The above effects might account for the improved efficacy of low-dose tolvaptan in patients during decompensation of liver function. To date, there is no simple endogenous marker to predict hepatic function with respect to the elimination capacity of specific drugs and guide dose adjustment in patients with liver injury. The semiquantitative Child-Pugh score is frequently used to assess the severity of liver function impairment. However, the Child-Pugh score only offers rough guidance for dosage adjustment and more sensitive markers need to be developed to guide drug dosage adjustment in patients with hepatic dysfunction. Finally, we cannot ignore that the concentration of serum sodium of this patient (about $130 \mathrm{mmol} / \mathrm{L}$ ) did not reach normal level during the tolvaptan therapy. Since hyponatremia develops slowly and cirrhotic patients show good hyponatremia tolerance, $130 \mathrm{mmol} / \mathrm{L}$ might be enough for the chronic dilutional hyponatremia patient.

The improvement of hyponatremia such as reduced occurrence of hepatic encephalopathy $[9,10]$ and improved quality of life [11] and prognosis of cirrhosis [12] may also lead to clinical benefits in patients. In our study, the hyponatremia correction was accompanied by gradual improvement of liver function and the Child-Pugh score. However, further studies are needed to investigate the clinical benefits of tolvaptan therapy after the correction of dilutional hyponatremia. In contrast, a recent meta-analysis indicated that the treatment of dilutional hyponatremia with vaptans did not result in a good prognosis. Twelve randomized, controlled trials with a total of 2,266 patients were included in this analysis, and the main outcome measures were mortality, spontaneous peritonitis, hepatic encephalopathy, and upper gastrointestinal hemorrhage. The results showed that vaptans could significantly increase serum sodium levels and lead to reduction in weight, whereas there was no clear difference between vaptans and placebo groups regarding prognosis [13]. During the therapy, we cannot ignore another phenomenon that although the urine volume was large, we still cannot decrease the dosage of diuretic or stop it during the therapy of tolvaptan. This may contribute to different mechanisms of drugs action.

The results of the present study suggested that, for cirrhotic patients with higher Child-Pugh scores (Class C) and serum sodium concentrations of $<120 \mathrm{mmol} / \mathrm{L}$, lowdose tolvaptan is effective for gradually increasing serum sodium concentrations, maintaining electrolyte balance, and possibly improving liver function. Further studies are needed to determine the optimal method of tolvaptan dose adjustment.

\section{Conflict of Interests}

The authors declare that there is no conflict of interests regarding the publication of this paper.

\section{Acknowledgments}

This study was supported by the Grant from the Medical and Science Plan of Zhejiang Province (2013KYB091).

\section{References}

[1] P. Angeli, F. Wong, H. Watson et al., "Hyponatremia in cirrhosis: results of a patient population survey," Hepatology, vol. 44, no. 6, pp. 1535-1542, 2006.

[2] C. C. Wu, L. K. Yeung, W. S. Tsai et al., "Incidence and factors predictive of acute renal failure in patients with advanced liver cirrhosis," Clinical Nephrology, vol. 65, no. 1, pp. 28-33, 2006.

[3] P. Ginès, F. Wong, H. Watson, S. Milutinovic, L. R. del Arbol, and D. Olteanu, "Effects of satavaptan, a selective vasopressin $V_{2}$ receptor antagonist, on ascites and serum sodium in cirrhosis with hyponatremia: a randomized trial," Hepatology, vol. 48, no. 1, pp. 204-213, 2008.

[4] L. Castello, M. Pirisi, P. P. Sainaghi, and E. Bartoli, "Hyponatremia in liver cirrhosis: pathophysiological principles of management," Digestive and Liver Disease, vol. 37, no. 2, pp. 73-81, 2005.

[5] R. W. Schrier, P. Gross, M. Gheorghiade et al., “Tolvaptan, a selective oral vasopressin $\mathrm{V}_{2}$-receptor antagonist, for hyponatremia," The New England Journal of Medicine, vol. 355, no. 20, pp. 2099-2112, 2006.

[6] I. Sakaida, M. Yanase, Y. Kobayashi, T. Yasutake, M. Okada, and K. Okita, "The pharmacokinetics and pharmacodynamics of tolvaptan in patients with liver cirrhosis with insufficient response to conventional diuretics: a multicentre, double-blind, parallel-group, phase III study," The Journal of International Medical Research, vol. 40, no. 6, pp. 2381-2393, 2012.

[7] J. A. Johnson and T. N. Livingston, "Differences between blacks and whites in plasma protein binding of drugs," European Journal of Clinical Pharmacology, vol. 51, no. 6, pp. 485-488, 1997.

[8] R. K. Verbeeck, "Pharmacokinetics and dosage adjustment in patients with hepatic dysfunction," European Journal of Clinical Pharmacology, vol. 64, no. 12, pp. 1147-1161, 2008.

[9] M. Guevara, M. E. Baccaro, A. Torre et al., "Hyponatremia is a risk factor of hepatic encephalopathy in patients with cirrhosis: a prospective study with time-dependent analysis," The American Journal of Gastroenterology, vol. 104, no. 6, pp. 1382-1389, 2009.

[10] M. Guevara, M. E. Baccaro, J. Ríos et al., "Risk factors for hepatic encephalopathy in patients with cirrhosis and refractory ascites: relevance of serum sodium concentration," Liver International, vol. 30, no. 8, pp. 1137-1142, 2010.

[11] E. Solà, H. Watson, I. Graupera et al., "Factors related to quality of life in patients with cirrhosis and ascites: relevance of serum sodium concentration and leg edema," Journal of Hepatology, vol. 57, no. 6, pp. 1199-1206, 2012.

[12] C. P. Marroni, A. B. de Mello Brandão, A. W. Hennigen et al., "MELD scores with incorporation of serum sodium and 
death prediction in cirrhotic patients on the waiting list for liver transplantation: a single center experience in southern Brazil," Clinical Transplantation, vol. 26, no. 4, pp. E395-E401, 2012.

[13] E. Dahl, L. L. Gluud, N. Kimer, and A. Krag, "Meta-analysis: the safety and efficacy of vaptans (tolvaptan, satavaptan and lixivaptan) in cirrhosis with ascites or hyponatraemia," Alimentary Pharmacology \& Therapeutics, vol. 36, no. 7, pp. 619-626, 2012. 


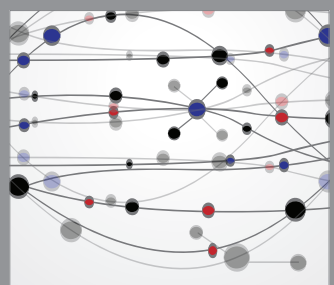

The Scientific World Journal
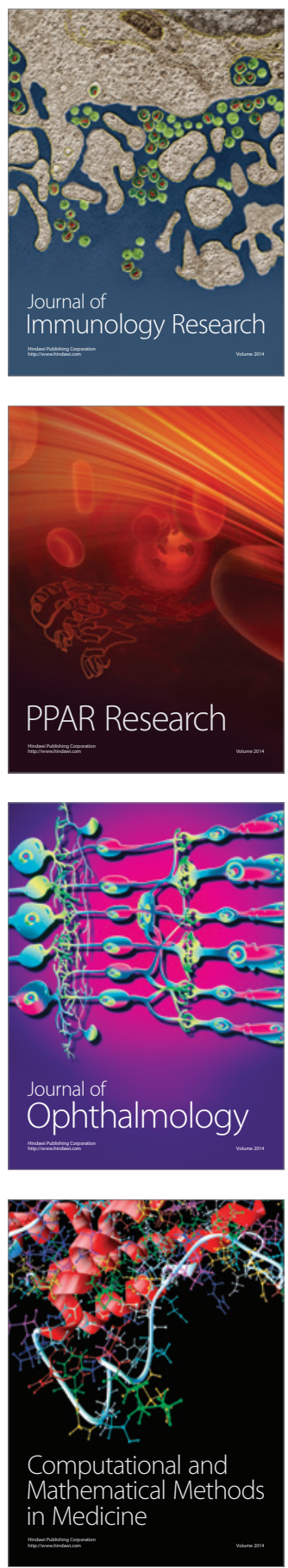

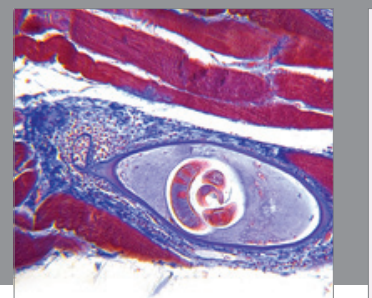

Gastroenterology

Research and Practice
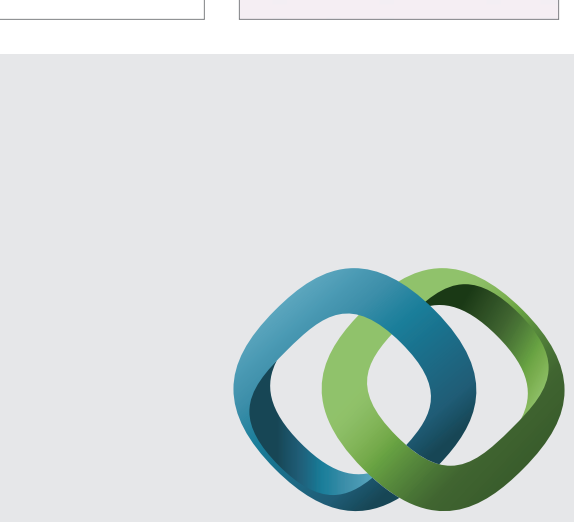

\section{Hindawi}

Submit your manuscripts at

http://www.hindawi.com
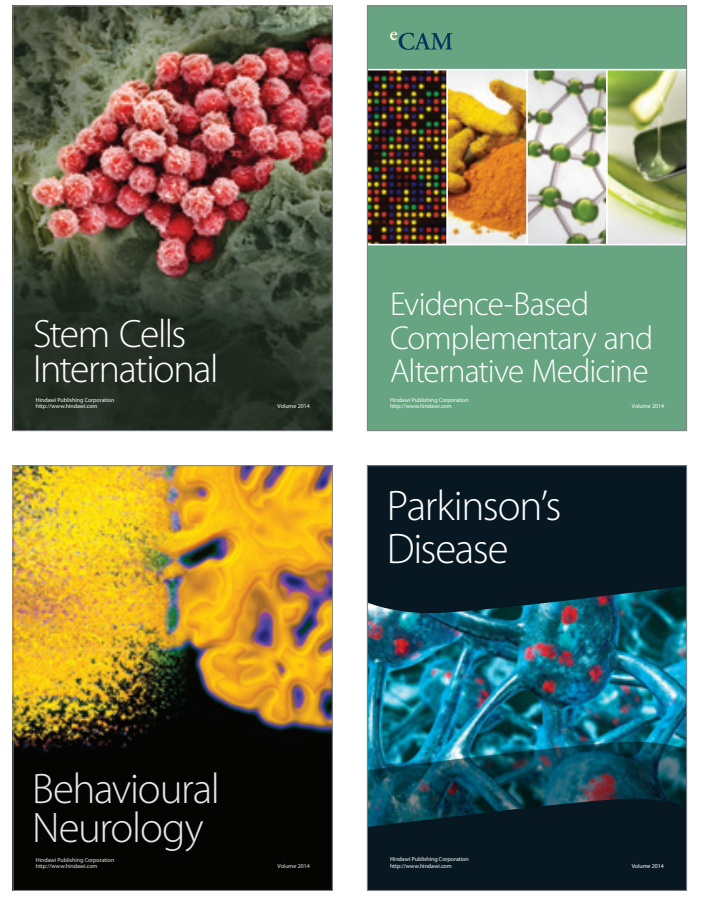
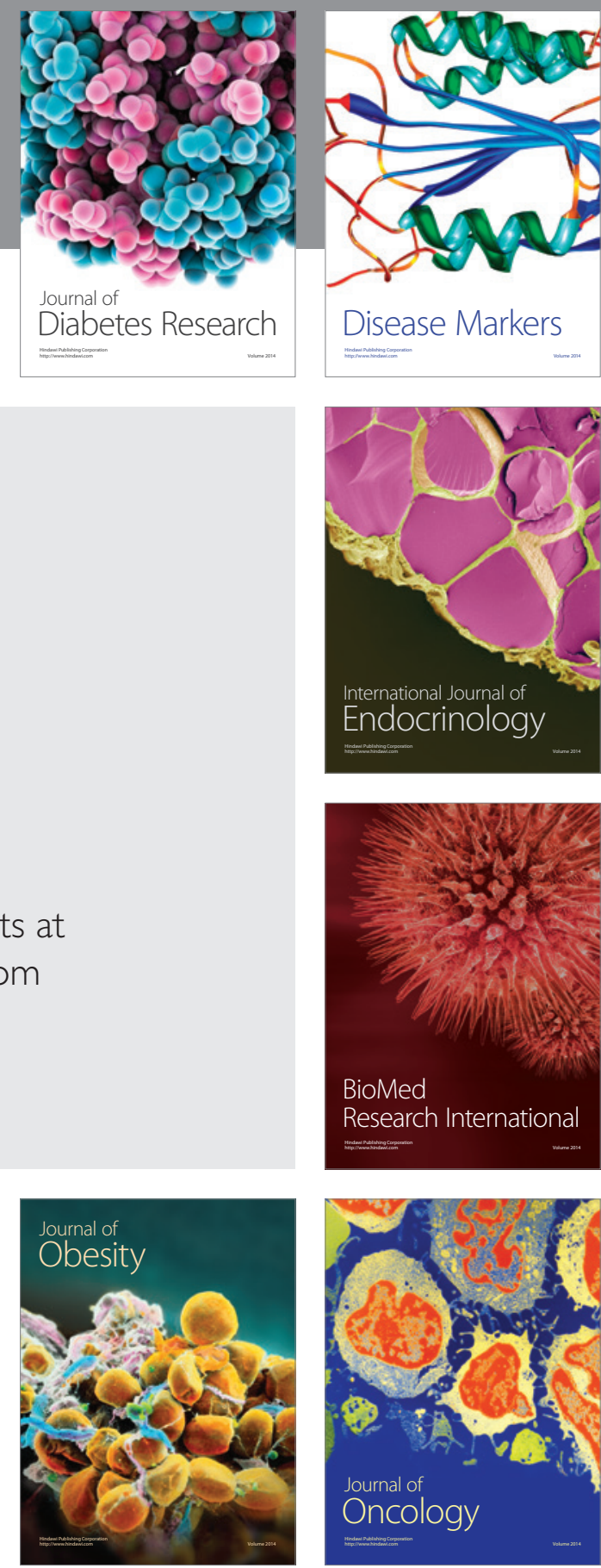

Disease Markers
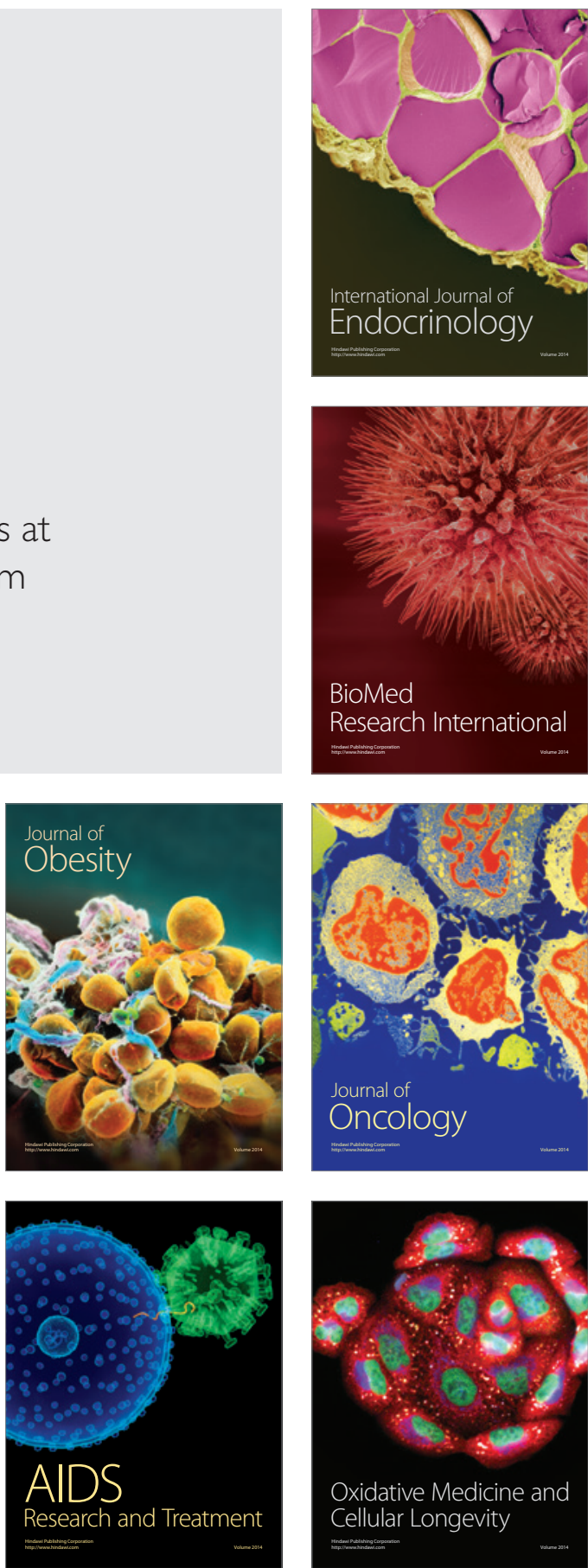